\title{
AGN types as different states of massive black holes
}

\author{
Boris V. Komberg \\ Astrospace Center, Physical Institute P. N. Lebedev, Moscow, Russian Federation \\ email: bkomberg@asc.rssi.ru
}

\begin{abstract}
After discovering microquasars - active stellar systems with relativistic outflows (Mirabel et al. 1992) - many papers bringing the observational arguments on similar nature of accreting systems with different $\mathrm{BH}$ masses (from stellar to galactic nuclei) have appeared. It became clear that a level of activity in such systems could depend on few parameters: $\mathrm{BH}$ mass and angular momentum; accretion rate and magnetization parameter of accretion disk $(\mathrm{AD})$; relative orientation of $\mathrm{BH}$ and $\mathrm{AD}$ angular momentum. The bolometric luminosity and characteristic variation time in such systems is proportional to BH masses, which span an interval in $5-8$ decades.
\end{abstract}

Keywords. Black hole physics - galaxies: nuclei - galaxies: active

\section{Introduction}

Active systems with different masses can be placed on a single sequence (Falcke \& Biermann 1996) that can be expressed using known data about Seyfert nuclei: $\log L_{5}(\mathrm{erg} / \mathrm{s}) \approx$ $1.67 \log L_{\mathrm{BBB}}(\mathrm{erg} / \mathrm{s})-33.4$. Here $L_{5}$ - radio luminosity at $5 \mathrm{GHz}, L_{\mathrm{BBB}}$ - disk luminosity in the big blue bump range. The more strict sequence connecting active stellar systems with different masses is traced on so called "fundamental plane" (Merloni et al. 2003): $\log L_{r}(\mathrm{erg} / \mathrm{s}) \approx 0,6 \log L_{\mathrm{XR}}(\mathrm{erg} / \mathrm{s})+0.78\left(M_{\mathrm{BH}} / M_{\odot}\right)+7.33$. Monitoring data on the brightest microQSOs in X-rays showed that light curves of such objects strongly vary in time which can be described by the existence of some characteristic states occasionally changing each other (Yadav 2001).

In the best-studied microQSO GRS 1915+105 sharp bursts of the soft X-ray radiation (spikes) occasionally appears in low/hard state followed by ejecting of radio outflows with relativistically moving components. A counter-rotation of the central $\mathrm{BH}$ and accreting material can also support jet formation (e.g. Koide et al. 2000).

Similar case is observed in some AGN (e.g. ON 231, 3C345; Belokon \& Babadzhanyats 2000) with VLBI jets appearing after bursts in the optical or UV range. But while in microQSOs X-ray-radio time lag is about hundreds of seconds, the observed time lag in the case of AGN is of order of years. Furthermore, it should be noted that mean AD temperature is connected with $\mathrm{BH}$ mass: $T_{\mathrm{D}}=2 \cdot 10^{7}\left(M_{\mathrm{BH}} / M_{\odot}\right)^{-1.4} \mathrm{~K}$. So X-ray bursts from disks of microQSOs correspond to UV bursts from AGN disks.

\section{AGN types as different states of massive black holes}

X-ray monitoring of microQSOs shows that they can be in the strong radio state for no more than 0.1 of time (e.g. Yadav 2001). It means that strong radio emission could be observed at 0.1 of microQSOs. This ratio looks similar to that of quasars - the fraction of the radio loud QSOs is $\sim 0.1$ from the radio quiet QSOs. This also refers to the ratio of the radio galaxies to the luminous E-galaxies (e.g. Komberg 2003; Nipoti et al. 2005). If these coincidences are not random, they can be the evidence of strong radio 
emission of AGN being relatively short state of its activity. And if radio quiet- radio loud ratio transition in microquasars lasts $10^{3} \mathrm{~s}$, in AGN it corresponds to $10^{2}-10^{3}$ yrs. Such transition has been confirmed by the detection of the extended steep spectrum radio structures at the long radio wavelengths $(90 \mathrm{~cm})$ of the radio quiet QSOs - the remains of the past nuclear activity (Blundel et al. 2000). Different AGN states are well observed in BL Lacertae objects. They have a typical "two-hump" spectral energy distribution (SED) $\log \nu f_{\nu}-\log \nu$ (Mei et al. 2002). A peak with a maximum on the lower frequency $\nu_{\mathrm{P} 1}$ (synchrotron peak) and that one with a maximum on the higher frequency $\nu_{\mathrm{P} 2}$ (inverse Compton peak) can move in the frequency axes depending on the radio luminosity: $\log L_{r}(\mathrm{erg} / \mathrm{s})=-0.67 \log \nu_{\mathrm{P} 1}+53.13$ (Di Mateo \& Psaltis 1999). Radio luminosity depends on the accretion rate increasing in low/hard state when $m<m_{\text {crit }}$. This shows a similarity in the accreting systems with very different $\mathrm{BH}$ masses.

Another common phenomena for the systems considered can be so called quasiperiodic oscillations (QPO) observed in microquasars in X-rays. Low-frequency QPO with $\nu_{\mathrm{QPO}}<10 \mathrm{~Hz}$ are frequently observed in the low/hard state, rarely in the intermediate state and never in the high/soft state. One point of view on QPO nature lies in the assumption of instabilities on the inner edge of the accretion disk $\left(r_{\mathrm{in}} \sim 10 R_{\mathrm{g}}\right)$ characterized by Keplerian frequency (e.g. Belanger et al. 2006): $\nu_{\mathrm{k}}=\frac{1}{2} \pi\left(G M_{\mathrm{BH}} / R^{3}\right)^{1 / 2}\left(M_{\mathrm{BH}}\right)^{-1}$. Observations give $\nu_{\mathrm{k}} \sim 0.1-10 \mathrm{~Hz}$ for microquasars. So for AGN with $M_{\mathrm{BH}} \sim 10^{6-9} M_{\odot}$ QPO frequencies correspond to typical oscillation times of order of days to months.

\section{Conclusions}

Observational arguments favoring to the similarity of different active system's properties listed above argue that different types of AGN are in fact the different states of massive accreting $\mathrm{BH}$ in the galactic centers. Conclusions following such assumption could promote a deeper understanding of AGN evolution. We can list some of them:

1. By the analogy with unstable "intermediate" state of microquasars, BL Lacertae objects can be thought to have a strongly variable intermediate state connecting the radio loud and radio quiet QSOs (RLQ and RQQ). In that case two types of BL Lacertae objects are possible. When transition is from RLQ to RQQ state, then RL BL Lac would be observed, and RQ BL Lac could be observed in opposite case.

2. One can expect higher accretion rates of $\mathrm{BHs}$ in the nuclei of the distant massive galaxies on large redshifts. That in turn leads to a smaller probability of the radio loud QSOs detection on higher $z$. So one should expect a reduction of RL QSO to RQ QSO number ratio on large $z$. Respectively, the RL BL Lac to RQ BL Lac number ratio, also should decrease on large $z$.

\section{References}

Mirabel, I. F., Rodrigez, L. F. \& Cordier, B. 1992, Nature, 358, 215

Falcke, H. \& Biermann, P. L. 1996, A\&A, 308, 321

Merloni, A., Heinz, S. \& Di Matteo, T. 2003, MNRAS, 345, 1057

Yadav, J. S. 2001, AJ, 548, 876

Koide, S. et al. 2000, AJ, 536, 668

Belokon, E. T. \& Babadzhanyats, M. K. 2000, A\&A, 356 , L21

Komberg, B. V. 2003, http://www.asc.rssi.ru (Educational Center, in Russian)

Nipoti, C., Blundell, K. M. \& Binney, J. 2005 MNRAS, 361, 633

Blundell, K. M. \& Rawkings, S. 2000, AJ, 562, L5

Mei, D. C., Zhang, L., \& Jiang, Z. J. 2002, A\&A, 391, 917

Di Mateo, T. \& Psaltis, D. 1999, AJ, 526, L101

Belanger, G., Terrier, R., De Jager, O., Goldwurm A. \& Melia, F. 2006, ApJL, submitted (astro$\mathrm{ph} / 0604337)$ 